\title{
Uniformização local: redução ao caso de valorizações de posto um
}

\author{
Michael Willyans Borges de Moraes
}



Assinatura:

\title{
Michael Willyans Borges de Moraes
}

\section{Uniformização local: redução ao caso de valorizações de posto um}

\begin{abstract}
Dissertação apresentada ao Instituto de Ciências Matemáticas e de Computação - ICMC-USP, como parte dos requisitos para obtenção do título de Mestre em Ciências - Matemática. EXEMPLAR $D E D E F E S A$
\end{abstract}

Área de Concentração: Matemática

Orientador: Prof. Dr. Daniel Levcovitz

USP - São Carlos

Julho de 2017 
Ficha catalográfica elaborada pela Biblioteca Prof. Achille Bassi e Seção Técnica de Informática, ICMC/USP, com os dados fornecidos pelo(a) autor(a)

Moraes, Michael Willyans Borges de
Uniformização local: redução ao caso de
valorizações de posto um / Michael Willyans Borges
de Moraes; orientador Daniel Levcovitz. - São Carlos
- SP, 2017.
38 p.
Dissertação (Mestrado - Programa de Pós-Graduação
em Matemática) - Instituto de Ciências Matemáticas e
de Computação, Universidade de São Paulo, 2017.
1. Uniformização local. 2. Valorizações.
3. Blowing up local. 4. Resolução de singularidades.
I. Levcovitz, Daniel, orient. II. Título.




\section{Michael Willyans Borges de Moraes}

\section{Local uniformization: reduction to the case of valuations of rank one}

Master dissertation submitted to the Instituto de Ciências Matemáticas e de Computação - ICMCUSP, in partial fulfillment of the requirements for the degree of the Master Program in Mathematics. EXAMINATION BOARD PRESENTATION COPY

Concentration Area: Mathematics

Advisor: Prof. Dr. Daniel Levcovitz

USP - São Carlos

July 2017 

Ao meu irmão Thomas Edison, in memorian 

Os agradecimentos principais são destinados ao meu orientador, Prof. Dr. Daniel Levcovitz, o professor mais querido do ICMC, que sempre me foi atencioso e amigo, e que tenho muito orgulho de o ter como orientador. E ao Dr. Josnei Novacoski, um dos autores do artigo em que esta dissertação é baseada, pelas várias horas que se dispôs a me ajudar a entender o tema, e por sempre me fazer buscar melhorar este trabalho.

Agradecimentos especiais são destinados a toda minha família, pelo suporte que sempre me deram. Também a todos os meus amigos, e ao Prof. Dr. Rogério Picanço, da Universidade Federal de Viçosa, que foi meu orientador e amigo na graduação, e que até hoje sempre me ajuda quando preciso. 

"As invenções são, sobretudo, o resultado de um trabalho de teimoso." (Santos Dumont) 



\section{RESUMO}

MORAES, M. W. B. DE. Uniformização local: redução ao caso de valorizações de posto um. 2017. 38 p. Dissertação (Mestrado em Ciências - Matemática) - Instituto de Ciências Matemáticas e de Computação, Universidade de São Paulo, São Carlos - SP, 2017.

Este trabalho trata da uniformização local, que é um passo do método de Zariski para provar resolução de singularidades em variedades algébricas. O método consiste numa abordagem por teoria de valorizações, e esta dissertação se baseia no artigo [NS], de Novacoski e Spivakovsky, que consiste em reduzir a prova da uniformização local para valorizações de qualquer posto, a provar apenas para os casos de posto um.

Palavras-chave: Uniformização local, Valorizações, Blowing up local, Resolução de singularidades. 



\section{ABSTRACT}

MORAES, M. W. B. DE. Local uniformization: reduction to the case of valuations of rank one. 2017. 38 p. Dissertação (Mestrado em Ciências - Matemática) - Instituto de Ciências Matemáticas e de Computação, Universidade de São Paulo, São Carlos - SP, 2017.

This work deals with local uniformization, which is a step in the method of Zariski to prove resolution of singularities for algebraic varieties. The method consists in an approach using valuation theory and this dissertation is based on the paper [NS], by Novacoski and Spivakovsky, which consists in reduce the proof of local uniformization for all cases to prove only the cases of rank one.

Keywords: Local uniformization, Valuations, Local blowing up, Resolution of singularities. 



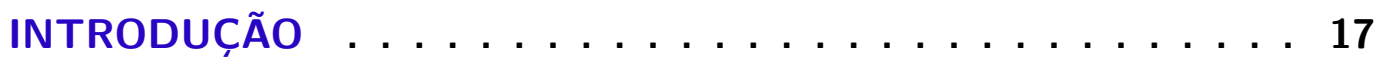

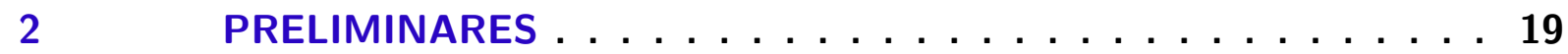

2.1 Convenções e fatos básicos . . . . . . . . . . . . . 19

2.2 Dimensão de Krull . . . . . . . . . . . . . . . . . . 19

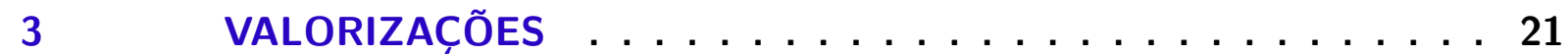

$3.1 \quad$ Anéis de valorização . . . . . . . . . . . . . . 21

3.2 Espectro de um anel de valorização . . . . . . . . . . . . . . 22

3.3 Grupos abelianos ordenados . . . . . . . . . . . . . . . 23

$3.4 \quad$ Valorizações . . . . . . . . . . . . . . . . . 23

$3.5 \quad$ Construindo valorizações . . . . . . . . . . . . . . . . . 24

3.6 Convexidade em grupos abelianos ordenados . . . . . . . . . . . 25

3.7 Decomposição de uma valorização . . . . . . . . . . . . . . . 26

4 EXTENSÕES CENTRADAS . . . . . . . . . . . . . 27

$4.1 \quad$ Centralização . . . . . . . . . . . . . . . 27

4.2 Extensões centradas em valorizações . . . . . . . . . . . . 28

$4.2 .1 \quad$ Caso $v^{\prime} \ldots \ldots \ldots \ldots \ldots \ldots \ldots \ldots$

$4.2 .2 \quad$ Caso $\bar{v} \ldots \ldots \ldots \ldots \ldots \ldots \ldots$

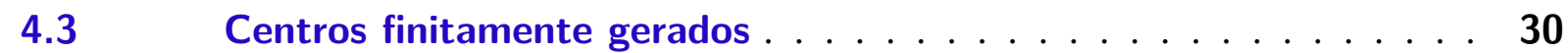

5 UNIFORMIZAÇÃO LOCAL . . . . . . . . . . . . . . . 33

$5.1 \quad$ Uniformização local . . . . . . . . . . . . . . . . 33

5.2 Uniformização local e decomposição de valorizações . . . . . . . . . 34

$5.3 \quad$ Dimensão e posto . . . . . . . . . . . . . . . 34

$5.4 \quad$ Redução a valorizações de posto um . . . . . . . . . . . . 35

REFERÊNCIAS . . . . . . . . . . . . . . . . . . . 37 



\section{1}

\section{INTRODUÇÃO}

O problema da uniformização local é um passo do método de Zariski para provar a existência de resolução de singularidades em variedades algébricas. Este problema lida com valorizações, e esta dissertação é baseada no artigo [NS], de Novacoski e Spivakovsky, que trata de reduzir o problema a casos aparentemente mais simples, os casos de valorizações de posto um.

Abaixo descreveremos, a grosso modo, como a uniformização local pode ser um passo para resolver singularidades. Alguns termos ficarão sem definição por estarem fora do foco da dissertação, outros as definições aparecerão no decorrer da dissertação.

Seja $X$ uma variedade (esquema) irredutível e reduzida sobre um corpo $K$ e $L \mid K$ seu corpo de funções associado, (a notação $L \mid K$ representa extensão de corpos). Dizemos que $X$ é um modelo para $L \mid K$. Por definição, um ponto de $X$ é um ideal primo $P$ de uma $K$-álgebra finitamente gerada $A$. O ponto $P$ é um ponto não-singular (ou regular) de $X$, se o anel $A_{P}$ é regular. Caso contrário, $P$ é singular. Uma resolução de singularidades para $X$ é um modelo $X^{\prime}$ para $L \mid K$, sem pontos singulares, com um morfismo birracional próprio $\pi: X^{\prime} \rightarrow X$.

Cada valorização $v$ em $L$, trivial em $K$, corresponde a um único ponto em $X$, denomidado de centro de $v$ em $X$. O problema da uniformização local resolvido para $v$ em $X$, nos dá um modelo $X(v)$ para $L \mid K$ e um morfismo birracional $\pi_{v}: X(v) \rightarrow X$, cujo centro de $v$ em $X(v)$ é regular. Este processo pode ser chamado de resolução local de singularides.

O conjunto de todas estas valorizações em $L$ e triviais $K$ é denotado por $S(L \mid K)$, e a função "tomar o centro"é uma função sobrejetiva em $X$. Em $S(L \mid K)$ é definido uma topologia que faz com que o conjunto das valorizações com centro regular em $X$, seja um conjunto aberto. Com esta topologia $S(L \mid K)$ é chamado de espaço de Riemann-Zariski, e é um espaço compacto. Graças a compacidade de $S(L \mid K)$ e ao fato de que o conjunto das valorizações com centro regular numa variedade é aberto, resolvendo o problema da uniformização local para toda valorização, podemos obter um conjunto finito de resoluções locais, de modo que qualquer valorização tenha 
centro regular em alguma destas resoluções locais. O passo final consiste em 'colar' as resoluções locais para obter uma resolução global.

Em 1940, em [Z], Zariski prova uniformização local para todos os casos em que o corpo base é de característica zero. Com este resultado, Zariski teve sucesso em colar as soluções locais e obter resolução de singularidades para variedades de dimensão até três. Em 1964, em $[\mathrm{H}]$, Hironaka prova resolução de singularidades para qualquer variedade sobre corpos de característica zero, sem usar esta abordagem por uniformização local. Hironaka foi premiado com a Medalha Fields por este resultado. Sobre corpos de característica positiva, os problemas da uniformização local e resolução de singularidades estão em abertos.

Em 1956, em [A1], Abhyankar prova uniformização local para superfícies sobre corpos de qualquer característica, e usando isto, em [A2], prova resolução de singularidades para superfícies em qualquer característica. Cossart e Piltant provam, em 2008, em [CP1] e [CP2], local uniformização e resolução de singularidades para variedades de dimensão três. Outro resultado obtido foi uniformização local para valorizações de Abhyankar, por Knaf e Kuhlmann, em [KK], em 2005.

Hoje, vários matemáticos trabalham no problema da uniformização local, em programas diferentes. Alguns exemplos são Knaf e Kuhlmann ([KK1] e [KK2]), usando teoria de ramificação, Spivakovsky ([SP]), usando teoria de blowing ups locais, e Teissier ([T1] e [T2]), usando geometria tórica. 
O objetivo deste capítulo é estabelecer as convenções e expor conceitos e resultados da álgebra que serão usados durante toda a dissertação. A principal ferramenta algébrica deste trabalho é a localização em domínios. Também usaremos conceitos de dimensão em domínios locais e o teorema da dimensão de Krull. Estes itens serão apresentados na segunda seção deste capítulo.

\subsection{Convenções e fatos básicos}

Sejam $A$ um domínio com corpo de frações $L$ e $P$ um ideal primo de $A$. A localização $A_{P}$, de $A$ em $P$, é o subanel $A_{P}:=\left\{\frac{a}{b} \mid a \in A, b \in R \backslash P\right\}$ de $L$. O anel $A_{P}$ é local (i.e. $A_{P}$ tem apenas um ideal maximal), com ideal maximal $P A_{P}$. Observe que se $\left\{\frac{x_{i}}{a_{i}}\right\}_{i \in I} \subseteq P A_{P}$ é um conjunto de geradores de $P A_{P}$, então também é o conjunto $\left\{x_{i}\right\}_{i \in I} \subseteq P$. Por outro lado, se $\left\{x_{i}\right\}_{i \in I} \subseteq P$ gera $P A_{P}$, então, para todo $x \in P$, se tem $a \in A \backslash P$ tal que $a x$ é combinação linear de elementos $\left\{x_{i}\right\}_{i \in I}$ em $P$ (basta obter $x$ como combinação linear de $\left\{x_{i}\right\}_{i \in I}$ em $A_{P}$, e depois multiplicar os dois membros da igualdade por $a$, de modo que a equação só envolva elementos de $A$ ).

Se $A^{\prime}$ é um subanel de $A$, então existe um mergulho natural $\overline{A^{\prime}}:=\frac{A^{\prime}}{A^{\prime} \cap P} \hookrightarrow \bar{A}:=\frac{A}{P}$. A fim de simplificar o texto, o anel $\overline{A^{\prime}}$ será sempre considerado um subanel de $\bar{A}$, ou seja, $\overline{A^{\prime}} \subseteq \bar{A}$, e um elemento $\bar{b} \in \bar{A}$ pertence a $\overline{A^{\prime}}$ se, e somente se, existe $a \in A^{\prime}$ tal que $\bar{a}=\bar{b}$.

\subsection{Dimensão de Krull}

Seja $A$ um domínio. Uma cadeia de ideais primos de $A$ é uma sequência de ideais primos de $A$ da forma $P_{0} \subset P_{1} \subset \cdots \subset P_{r} \operatorname{com} P_{i} \neq P_{i+1}$ para $i=0, \cdots, r-1$. Os ideais $P_{0}$ e $P_{r}$ são chamados de origem e extremidade da cadeia, respectivamente. Dizemos que o comprimento da cadeia acima é $r$. A dimensão de Krull de $A$, ou simplesmente dimensão de $A$, denotada por $\operatorname{dim} A$, é definida como sendo o supremo do conjunto dos comprimentos de cadeias de ideais 
primos de $A$, podendo ser finita ou infinita. Se $P$ é um ideal primo de $A$, definimos a altura de $P$, denotada por $h t(P)$, como sendo o supremo de comprimentos de cadeias de ideais primos de $A$ com extremidade $P$.

A proposição abaixo nos dá um conjunto de propriedades básicas de dimensão e altura. A prova destes fatos podem ser vistas no capítulo III de [S].

Proposição 2.1. São válidas as seguintes propriedades:

(i) $h t(P)=\operatorname{dim} A_{P}$;

(ii) $h t(P)+\operatorname{dim} A / P \leq \operatorname{dim} A$;

(iii) Se $P^{\prime}$ é ideal primo de $A$ tal que $P^{\prime} \subseteq P$, então $h t\left(P^{\prime}\right) \leq h t(P)$;

(iv) $\operatorname{Se}(A, M)$ é local, então $\operatorname{dim} A=h t(M)$;

(v) Se $(A, M)$ é local, $\operatorname{dim} A$ é finita e $P \neq M$, então $\operatorname{dim} A_{P}<\operatorname{dim} A$.

Sejam $A$ um domínio noetheriano (i.e., todos seus ideais são finitamente gerados) e $I$ um ideal de $A$. Denotamos por $\mu(I)$ o número natural que corresponde a menor cardinalidade possível de um conjunto de geradores de $I$. Dizemos que um domínio noetheriano local $(A, M)$ é regular se $\operatorname{dim} A=\mu(M)$. O teorema abaixo é conhecido como teorema da dimensão de Krull ([N], Teorema 9.3).

Teorema 2.2. Se $(A, M)$ é um domínio noetheriano local, então $\operatorname{dim} A \leq \mu(M)$.

Note que se $(A, M)$ é um domínio local e $P$ é um ideal primo de $A$, então $A / P$ é domínio local com ideal maximal $M / P$. A proposição abaixo será importante nos nossos resultados finais.

Proposição 2.3. Sejam $(A, M)$ um domínio noetheriano local e $P$ um ideal primo de $A$. Se $A / P$ é regular e $\mu(P) \leq \operatorname{dim} A_{P}$, então $A$ é regular.

Demonstração. Sejam $r:=\operatorname{dim} A_{P}$ e $s:=\operatorname{dim} A / P=\mu(M / P)$. Observe que, se $a_{1}, \cdots, a_{r}$ geram $P$ e $\bar{b}_{1}, \cdots, \bar{b}_{s}$ geram $M / P$ (tais geradores existem pelas hipóteses), então $a_{1}, \cdots, a_{r}, b_{1}, \cdots, b_{s}$ geram $M$, e $\log o r+s \geq \mu(M)$. Pelos itens (ii) e (i) da proposição 1.1, temos $\operatorname{dim} A \geq \operatorname{dim} A_{P}+$ $\operatorname{dim} A / P=r+s$, ou seja $\operatorname{dim} A \geq r+s \geq \mu(M)$. O teorema da dimensão de Krull nos dá a desigualdade contrária, e assim $\operatorname{dim} A=\mu(M)$ e $A$ é regular. 
Um anel de valorização $\mathscr{O}$ é precisamente um domínio em que seus ideais principais estão totalmente ordenados pela relação de inclusão. Esta propriedade nos induz a definir uma função de $\mathscr{O}$ em um conjunto ordenado, com imagem de $x$ maior que a imagem de $y$ se, e somente se, $(x) \varsubsetneqq(y)$, onde $x, y \in \mathscr{O}$. Esta função é naturalmente estendida para $\operatorname{Frac}(\mathscr{O})$, e a chamamos de valorização. $\mathrm{O}$ conjunto ordenado tem estrutura de grupo abeliano ordenado, e propriedades de $\mathscr{O}$ estão relacionadas com propriedades deste grupo.

Deixaremos de mencionar alguns fatos básicos sobre valorizações que não terão utilidade nesta dissertação. Porém, para o problema da uniformização local, um conhecimento vasto deste tema é necessário, e recomendamos $[\mathrm{EP}]$ e [V].

\subsection{Anéis de valorização}

Um subanel $\mathscr{O}$ de um corpo $L$ é um anel de valorização de $L$ se, para todo $x \in L^{\times}$, temos $x \in \mathscr{O}$ ou $x^{-1} \in \mathscr{O}$. Dizemos que um domínio $\mathscr{O}$ é um anel de valorização, sem mencionar um corpo, se este é um anel de valorização de seu corpo de frações.

Exemplo 3.1. Seja $K[X]$ o anel de polinômios em uma variável sobre o corpo $K$ e $p \in K[X]$ um polinômio irredutível. A localização $K[X]_{(p)}$ é um anel de valorização de $K(X)$. Também, $\mathbb{Z}_{(p)}$ é um anel de valorização de $\mathbb{Q}$, onde $p$ é um número primo.

O conjunto de todos os ideais de um anel de valorização é totalmente ordenado pela relação de inclusão. De fato, sejam $I$ e $J$ ideais de um anel de valorização $\mathscr{O}$ de $L$, e digamos que exista $x \in J \backslash I$. Então, para todo $y \in I$, não podemos ter $x / y \in \mathscr{O}, \operatorname{logo} y / x \in \mathscr{O}$ e assim $y=x .(y / x) \in J$, e portanto $I \subset J$. Reversamente, se $A$ é um domínio onde o conjunto de todos seus ideais principais é totalmente ordenado pela relação de inclusão, então $A$ é um anel de 
valorização. De fato, se $x, y \in A$ são não nulos, devemos ter $(x) \subseteq(y)$ ou $(y) \subseteq(x)$, e se por exemplo tivermos $(x) \subseteq(y)$, existe $a \in A$ tal que $x=y a$, e $\log \frac{x}{y}=a \in A$.

Pelo que foi dito acima, todo anel de valorização é anel local. Em geral, usaremos $\mathscr{O}$ 's para representar anéis de valorização, e $m$ 's para representar seus ideais maximais.

Seja $L$ um corpo. Se $(A, M)$ e $\left(A^{\prime}, M^{\prime}\right)$ são dois subanéis locais de $L$, dizemos que $(A, M)$ é dominado por $\left(A^{\prime}, M^{\prime}\right)$ se $A \subseteq A^{\prime}$ e $M=A \cap M^{\prime}$. O teorema da extensão de Chevalley ([EP], Teorema 3.1.1) nos diz que, dado $(A, M) \subseteq L$, existe um anel de valorização de $L$ que domina $(A, M)$. Observe que um anel de valorização de $L$ não pode ser dominado por outro anel de valorização de $L$. Portanto, um anel de valorização de $L$ pode ser caracterizado também como um elemento maximal do conjunto dos anéis locais com corpo de fração $L$ com a ordem dada pela relação de dominação. Temos provada então a proposição abaixo:

Proposição 3.2. Um subanel $\mathscr{O}$ de um corpo $L$ é um anel de valorização de $L$ se satisfaz alguma das condições equivalentes abaixo:

(i) Para todo $x \in L^{\times}$, temos $x \in \mathscr{O}$ ou $x^{-1} \in \mathscr{O}$.

(ii) $\mathrm{O}$ conjunto dos ideais de $\mathscr{O}$ é totalmente ordenado pela relação de inclusão.

(iii) $\mathrm{O}$ conjunto dos ideais principais de $\mathscr{O}$ é totalmente ordenado pela relação de inclusão.

(iv) $(\mathscr{O}, m)$ é elemento máximo pela relação de dominação nos domínios locais contidos em $L$.

Observação 3.3. Se $K$ é um subcorpo do corpo $L$ e $\mathscr{O}$ um anel de valorização de $L$, então $K \cap \mathscr{O}$ é um anel de valorização de $K$.

\subsection{Espectro de um anel de valorização}

Sejam $(\mathscr{O}, m)$ um anel de valorização de $L$ e $P$ um ideal primo de $\mathscr{O}$. A localização $\mathscr{O}_{P}$ é anel de valorização de $L$ e contém $\mathscr{O}$. O ideal maximal $P \mathscr{O}_{P}$ de $\mathscr{O}_{P}$ é o próprio $P$. De fato, se tivessemos $a \in P \mathscr{O}_{P} \backslash P$, então $a^{-1} \notin \mathscr{O}_{P}$, que implica $a^{-1} \notin \mathscr{O}$, que implica $a \in \mathscr{O} \backslash P$. Logo $a$ deve ser inversível em $\mathscr{O}_{P}$, e assim não podemos ter $a \in P \mathscr{O}_{P}$.

Reversamente, se $(\mathscr{O}, m)$ e $\left(\mathscr{O}^{\prime}, m^{\prime}\right)$ são anéis de valorização de $L$ com $\mathscr{O} \subseteq \mathscr{O}^{\prime}$, então $m^{\prime} \subseteq m$ é um ideal primo de $\mathscr{O}$ e $\mathscr{O}^{\prime}=\mathscr{O}_{m^{\prime}}$. Chamamos o corpo $\mathscr{O} / m$ de corpo residual de $\mathscr{O}$, e o denotamos por $\bar{L}$. O anel local $\left(\mathscr{O} / \mathrm{m}^{\prime}, \mathrm{m} / \mathrm{m}^{\prime}\right)$ é anel de valorização do corpo residual $\bar{L}^{\prime}$ de $\mathscr{O}^{\prime}$. Escrevemos simplesmente $(\overline{\mathscr{O}}, \bar{m})$. Um par como $\mathscr{O}^{\prime}$ e $\overline{\mathscr{O}}$ formam uma decomposição de $\mathscr{O}$. A imagem inversa de $\overline{\mathscr{O}}$ pela projeção $\mathscr{O}^{\prime} \rightarrow \bar{L}^{\prime}$ é $\mathscr{O}$, devido a $m^{\prime} \subset \mathscr{O}$. Logo, cada anel de valorização de $\bar{L}^{\prime}$ corresponde a um único anel de valorização de $L$ contido em $\mathscr{O}^{\prime}$, e o contrário.

O espectro de um anel de valorização $\mathscr{O}$ de $L$ é totalmente ordenado pela relação de inclusão, e pelo que foi dito acima, os subanéis de $L$ que contém $\mathscr{O}$ também estão totalmente ordenados pela inclusão.

O posto de um anel de valorização $(\mathscr{O}, m)$ de $L$ é a dimensão de Krull de $\mathscr{O}$. Se o posto de $\mathscr{O}$ é finito, digamos $r$, e $P \subseteq m$ é o ideal primo de $\mathscr{O}$ de altura $s \leq r$, então o posto de $\mathscr{O}_{P}$ é $s$, e 
o posto do anel de valorização $\overline{\mathscr{O}}$ do corpo residual $\bar{L}_{P}$ de $\mathscr{O}_{P}$ é $r-s$.

Exemplo 3.4. Se $K[X]$ e $p$ são como no exemplo 3.1, o corpo residual de $K[X]_{(p)}$ é $K[X] /(p)$ (localização comuta com quociente). Observe que o posto de $K[X]_{(p)}$ é um, pois este só tem um ideal primo não nulo, a saber, $(p) K[X]_{(p)}$.

Exemplo 3.5. Considere os seguintes anéis de valorização: $\mathscr{O}^{\prime}:=K(Y)[X]_{(X)}$ de $L:=K(X, Y)$ e $\overline{\mathscr{O}}:=K[Y]_{(Y)}$ de $K(Y)$. Observe que o corpo residual $\bar{L}^{\prime}$ de $\mathscr{O}^{\prime}$ é isomorfo a $K(Y)$. Portanto, $\mathscr{O}^{\prime}$ e $\overline{\mathscr{O}}$ nos dão um anel valorização em $K(X, Y)$ de posto dois, pela imagem inversa de $\overline{\mathscr{O}}$ por $\mathscr{O}^{\prime} \rightarrow \bar{L}^{\prime}$.

\subsection{Grupos abelianos ordenados}

Antes de falarmos da função valorização, vamos definir grupo abeliano ordenado, que virá a ser a estrutura do grupo de valores da valorização.

Seja $\Gamma$ um grupo abeliano e $\leq$ uma ordem total em $\Gamma$. Dizemos que $(\Gamma, \leq)$ é um grupo abeliano ordenado se, para todos $a, b, c \in \Gamma, a \leq b$ implica $a+c \leq b+c$. Observe que um subgrupo de um grupo abeliano ordenado é um grupo abeliano ordenado com a ordem induzida.

Seja $(\Gamma, \leq)$ um grupo abeliano ordenado. Podemos considerar o conjunto $\Gamma \cup\{\infty\}$, onde $\infty$ é um símbolo, e estender a operação de $\Gamma$ dizendo que $a+\infty=\infty+a=\infty+\infty=\infty$. Estendemos também a ordem, dizendo que $a<\infty$ para todo $a \in \Gamma$. Dizemos que $(\Gamma \cup\{\infty\}, \leq)$ é um grupo abeliano ordenado estendido, considerando a operação e ordem estendidas como acima. Escreveremos apenas $\Gamma \cup\{\infty\}$ quando não há chance de confusão, e quando escrevermos $\Gamma \cup\{\infty\}$ já estaremos considerando o grupo abeliano ordenado estendido de $\Gamma$.

Um isomorfismo de grupos abelianos ordenados é um isomorfismo de grupos que preserva ordem. $\mathrm{O}$ símbolo $\simeq$ denotará tal tipo de equivalência isomorfica, quando fizer sentido.

Exemplo 3.6. O grupo $\mathbb{Z}$ é grupo abeliano ordenado com a ordem usual.

Exemplo 3.7. Se $\Gamma$ é um grupo abeliano ordenado, então também é $\Gamma \times \Gamma$ com a ordem lexográfica, que é a ordem dada por $(a, b) \leq\left(a^{\prime}, b^{\prime}\right)$ se, e somente se, $a<a^{\prime}$ ou $a=a^{\prime}$ e $b \leq b^{\prime}$. Escrevemos $(\Gamma \times \Gamma)_{\text {lex }}$ para significar o grupo $\Gamma \times \Gamma$ com esta ordem.

\subsection{Valorizações}

Seja $L$ um corpo. Uma valorização em $L$ é uma função sobrejetora $v: L \rightarrow \Gamma \cup\{\infty\}$, onde $\Gamma$ é um grupo abeliano ordenado, que satisfaz as condições

$$
\begin{gathered}
v(a)=\infty \text { implica } a=0 \\
v(a b)=v(a)+v(b) \\
v(a+b) \geq \min \{v(a), v(b)\} .
\end{gathered}
$$


Note que $v(1)=0$ e $v\left(a^{-1}\right)=-v(a)$, se $a \in L^{\times}$. Também, se $v(a)<v(b)$, então $v(a+$ $b)=v(a)$. O conjunto $\mathscr{O}_{v}:=\{a \in L \mid v(a) \geq 0\}$ é um anel de valorização de $L$ com ideal maximal $m_{v}:=\{a \in L \mid v(a)>0\}$. Observe que $\Gamma \simeq L^{\times} / \mathscr{O}_{v}^{\times}$como grupos abelianos. As notações $\mathscr{O}_{v} \mathrm{e}$ $m_{v}$, com índices $v$, são usadas para representar o anel de valorização $\left(\mathscr{O}_{v}, m_{v}\right)$ de $L$ relacionado à valorização $v$ pela definição acima, assim como $\bar{L}_{v}$ para representar o corpo residual $\mathscr{O}_{v} / m_{v}$.

Sejam $L$ um corpo e $\mathscr{O}$ um anel de valorização de $L$. O grupo abeliano $L^{\times} / \mathscr{O}^{\times}$com a ordem $\bar{a} \leq \bar{b}$ se, e somente se, $\frac{b}{a} \in \mathscr{O}$, onde $a, b \in L^{\times}$, é um grupo abeliano ordenado. A aplicação $v: L \rightarrow L^{\times} / \mathscr{O}^{\times} \cup\{\infty\}$, definida como $\left.v\right|_{L^{\times}}$sendo a projeção e $v(0)=\infty$, é uma valorização de $L$ $\operatorname{com} \mathscr{O}_{v}=\mathscr{O}$.

Como $v(a) \leq v(b)$ implica $\frac{b}{a} \in \mathscr{O}_{v}$ para qualquer valorização, valorizações e anéis de valorização estão relacionados biunivocamente, a menos de isomorfismo de grupos abelianos ordenados.

Exemplo 3.8. Sejam $K[X]$ e $p$ como no exemplo 3.1. A valorização $p$-ádica de $K(X)$ é a valorização $v_{p}: K(X) \rightarrow \mathbb{Z} \cup\{\infty\}$ que leva $f \in K[X]^{\times}$no numero natural que corresponde a maior potência de $p$ que divide $f$, e $\frac{f}{g} \in K(X)^{\times}$em $v_{p}(f)-v_{p}(g)$. Observe que $\mathscr{O}_{v_{p}}=\left\{\frac{f}{g} \in K(X) \mid p \nmid g\right\}$, que é precisamente a localização $K[X]_{(p)}$.

Observação 3.9. Sejam $v$ uma valorização no corpo $K$ e $L \mid K$ uma extensão de corpos. Pelo Teorema da extensão de Chevalley, existe uma valorização $w$ em $L$ que estende $v$. Temos uma imersão natural $K^{\times} / \mathscr{O}_{v}^{\times} \hookrightarrow L^{\times} / \mathscr{O}_{w}$, e logo o grupo de valores de $v$ pode ser visto como um subgrupo do grupo de valores de $w$.

Se $L \mid K$ é uma extensão de corpos e $v$ uma valorização em $L$, dizemos que $v$ é trivial em $K$ se $v\left(K^{\times}\right)=\{0\}$, ou equivalentemente, $K \subseteq \mathscr{O}_{v}$.

\subsection{Construindo valorizações}

O objetivo desta seção é adquirir ferramentas para construir valorizações de maneira mais fácil. Vamos começar enunciando um teorema que é provado em [EP] (Teorema 2.2.1 em $[\mathrm{EP}])$.

Teorema 3.10. Sejam $K$ um corpo, $\Gamma$ um subgrupo ordenado do grupo ordenado $\Gamma^{\prime}, v: K \rightarrow$ $\Gamma \cup\{\infty\}$ uma valorização, $\gamma \in \Gamma^{\prime}$, e $K[X]$ o anel de polinômios em uma variável sobre $K$. Para $f=\sum_{i=0}^{n} a_{i} X^{i} \in K[X]^{\times}$, defina

$$
w(f):=\min _{0 \leq i \leq n}\left\{v\left(a_{i}\right)+i \gamma\right\}
$$

$w(0)=0$ e, para $\frac{f}{g} \in K(X), w\left(\frac{f}{g}\right)=w(f)-w(g)$. Assuma que $\Gamma^{\prime}$ é gerado por $\Gamma \cup\{\gamma\}$. As equações acima definem a valorização $w: K(X) \rightarrow \Gamma^{\prime} \cup\{\infty\}$ em $K(X)$ que estende $v$. 
Observe que aplicando o teorema de forma iterada, podemos obter valorizações em $K\left[X_{1}, \cdots, X_{n}\right]$ que sejam extensões de valorizações em $K$.

Exemplo 3.11. Consire $\Gamma=(\mathbb{Z} \times \mathbb{Z})_{\text {lex }}$ e $K[X, Y]$ o anel de polinômios em duas variáveis sobre o corpo $K$. A valorização $v: K(X, Y) \rightarrow \Gamma \cup\{\infty\}$ definida por $v\left(K^{\times}\right)=\{0\}, v(Y)=(1,0)$ e $v(X)=(0,1)$ é uma extensão da valorização $X$-ádica em $K(X)$, que por sua vez é uma extensão da valorização trivial em $K$.

Daremos abaixo um exemplo e mostraremos em seguida que temos de fato uma valorização.

Exemplo 3.12. Considere o anel de polinômios $K[X, Y]$ e o polinômio irredutírvel $Y^{2}-X^{3}$. Considere $K[x, y]:=K[X, Y] /\left(Y^{2}-X^{3}\right)$, onde $x$ e $y$ são as classes de $X$ e $Y$ respctivamente. Defina $v: K(x, y) \rightarrow\left(\mathbb{Z}+\frac{3}{2} \mathbb{Z}\right) \cup\{\infty\}$, onde $\mathbb{Z}+\frac{3}{2} \mathbb{Z} \subset \mathbb{Q}$ com a ordem usual, $v\left(K^{\times}\right)=\{0\}$, $v(x)=1, v(y)=\frac{3}{2}$ e estendida para todo o domínio de maneira óbvia. $v$ é uma valorização em $K(x, y)$.

No exemplo acima, $K(x, y) \mid K(x)$ é uma extensão algébrica com o polinômio irredutível de $y$ sendo $X^{2}-x^{3}$. Em $K(x)$, temos a valorização $x$-ádica, e esta é extendida (e pode, pelo Teorema de Chevalley) para $K(x, y)$. Como $y^{2}-x^{3}=0$, temos $v\left(y^{2}-x^{3}\right)=\infty$, que implica $2 v(y)=3 v(x)$. Logo, o grupo de valores de $v$ deve conter $\mathbb{Z}+\frac{3}{2} \mathbb{Z}$. Para ver que já temos definida toda a valorização, veja que se $f$ é um polinômio em $K(x)[y]$, então este pode ser escrito por $f=\left(y^{2}-x^{3}\right) g+(y+a)$, onde $g \in K(x)[y]$. Temos então $v(f)=v(y+a)$. Se $y+a=0$, temos $v(f)=\infty$. Se $y+a \neq 0$ temos $v(y+a)=\min \{v(y), v(a)\}$. Portanto o grupo de valores de $v$ é $\mathbb{Z}+\frac{3}{2} \mathbb{Z}$ e $v$ é uma valorização.

\subsection{Convexidade em grupos abelianos ordenados}

Seja $\Gamma$ um grupo abeliano ordenado. Se $\gamma \in \Gamma$ é positivo, denotamos por $[-\gamma, \gamma]$ o conjunto $\{\alpha \in \Gamma \mid-\gamma \leq \alpha \leq \gamma\}$. Um subconjunto convexo de $\Gamma$ é um subconjunto de $\Gamma$ que é união de conjuntos da forma $[-\gamma, \gamma]$. Um subgrupo convexo de $\Gamma$ é um subgrupo que é subconjunto convexo de $\Gamma$. O conjunto dos subgrupos convexos de $\Gamma$ é totalmente ordenado pela relação de inclusão. O posto de $\Gamma$ é a cardinalidade do conjunto de todos os subgrupos convexos não nulos de $\Gamma$, podendo ser finito ou infinito.

Sejam $\Gamma$ um grupo abeliano ordenado e $\Delta$ um subgrupo convexo de $\Gamma$. O grupo quociente $\Gamma / \Delta$ é um grupo abeliano ordenado com ordem induzida por $\Gamma$ da seguinte forma: se $\alpha+\Delta, \beta+$ $\Delta \in \Gamma / \Delta$ não são iguais, e $\alpha<\beta$, então $\alpha+\Delta<\beta+\Delta$. Tal relação independe da escolha dos representantes. O posto de $\Gamma / \Delta$ é igual ao posto de $\Gamma$ menos o posto de $\Delta$.

Sejam $L$ um corpo, $\Gamma$ um grupo abeliano ordenado, $\Delta$ um subgrupo convexo de $\Gamma$ e $v: L \rightarrow$ $\Gamma \cup\{\infty\}$ uma valorização em $L$. É fácil verificar que o conjunto $P=\{a \in L \mid v(a)>\delta, \forall \delta \in \Delta\}$ 
é um ideal primo de $\mathscr{O}_{v}$. Dizemos que $P$ é o ideal primo correspondente a $\Delta$. Reversamente, se $P$ é um ideal primo de $\mathscr{O}_{v}$, então $\bigcup_{a \in \mathscr{O}_{v} \backslash P}[-v(a), v(a)]$ é subgrupo convexo de $\Gamma$. Observe que, se $\Delta_{1}$ e $\Delta_{2}$ são subgrupos convexos distintos de $\Gamma$ com ideais primos correspondentes $P_{1}, P_{2} \subset \mathscr{O}_{v}$ respectivamente, então $P_{1} \neq P_{2}$ e $\Delta_{1} \subset \Delta_{2}$ implica $P_{1} \supset P_{2}$. O posto de $\mathscr{O}_{v}$ é igual ao posto de $\Gamma$.

Observação 3.13. Se $\Gamma^{\prime}$ é um subgrupo de $\Gamma$, não necessariamente convexo, então o posto de $\Gamma^{\prime}$ é menor ou igual ao posto de $\Gamma$. Logo, a restrição de uma valorização nunca pode ter posto maior que o posto da inicial.

Exemplo 3.14. O grupo abeliano ordenado $(\mathbb{Z} \times \mathbb{Z})_{l e x}$ tem posto dois, e seus subgrupos convexos não nulos são $\{0\} \times \mathbb{Z}$ e o próprio $(\mathbb{Z} \times \mathbb{Z})_{\text {lex }}$.

\subsection{Decomposição de uma valorização}

Sejam $L$ um corpo, $\Gamma$ um grupo abeliano ordenado e $v: L \rightarrow \Gamma \cup\{\infty\}$ uma valorização em $L$. Sejam $\Delta$ um subgrupo convexo de $\Gamma$ e $P \subset \mathscr{O}_{v}$ seu ideal primo correspondente. Podemos considerar a valorização $v^{\prime}: L \rightarrow \Gamma / \Delta \cup\{\infty\}$, compondo $v$ com a projeção $\Gamma \rightarrow \Gamma / \Delta$. Temos $\mathscr{O}_{v^{\prime}}=\mathscr{O}_{v} \cup\left\{a \in L \backslash \mathscr{O}_{v} \mid v(a) \in \Delta\right\}$, que é precisamente a localização $\left(\mathscr{O}_{v}\right)_{P}$, e $m_{v^{\prime}}=P$.

Seja $\bar{L}_{v^{\prime}}=\mathscr{O}_{v^{\prime}} / m_{v^{\prime}}$ o corpo residual da valorização $v^{\prime}$ definida acima. Podemos considerar a função $\bar{v}: \bar{L}_{v^{\prime}} \rightarrow \Delta \cup\{\infty\}$ fazendo $\bar{v}(\bar{a})=v(a)$ se $\bar{a} \neq \overline{0}$, e $v(\overline{0})=\infty$. Devido a $v(a)<v(b)$, para todos $b \in m_{v^{\prime}}$ e $a \in L \backslash m_{v^{\prime}}$, temos que $v\left(a+m_{v^{\prime}}\right)=v(a)$, para todos $a \in L \backslash m_{v^{\prime}}$, e portanto $\bar{v}$ não depende do representante. A função $\bar{v}$ é uma valorização em $\bar{L}_{v^{\prime}}$. O anel de valorização correspondente a $\bar{v}$ é $\mathscr{O}_{\bar{v}}=\overline{O_{v}}=O_{v} / m_{v^{\prime}}$, com ideal maximal $m_{\bar{v}}=\overline{m_{v}}=m_{v} / m_{v^{\prime}}$.

O par $v^{\prime}$ e $\bar{v}$ acima é uma decomposição de $v$, e escrevemos $v=v^{\prime} \circ \bar{v}$. O posto de $v$ é igual a soma dos postos de $v^{\prime}$ e $\bar{v}$. Dizemos que a decomposição acima é trivial se $\mathscr{O}_{v}=\mathscr{O}_{v^{\prime}}$. Observe que se $v$ tem posto finito, por indução, podemos decompor $v$ em, possivelmente mais que duas, valorizações de posto um.

Exemplo 3.15. Considere a valorização $v$ do exemplo 3.11. Sejam $\Gamma:=(\mathbb{Z} \times \mathbb{Z})_{\text {lex }}, \Delta:=$ $\{0\} \times \mathbb{Z} \simeq \mathbb{Z}$ e $\Gamma / \Delta \simeq \mathbb{Z}$. Temos $v\left(K(X)^{\times}\right) \subseteq \Delta$, e $v(Y)=(1,0)$, que projetado em $\Gamma / \Delta$ é 1 . Portanto a valorização $v^{\prime}$ é a valorização $Y$-ádica em $K(X)(Y)$. Como o corpo residual de $v^{\prime}$ é $K(X)[Y] /(Y) \simeq K(X)$, e a valorização $\bar{v}$ é tal que $\bar{v}\left(K^{\times}\right)=\{0\}$ e $\bar{v}(Y)=1, \bar{v}$ é a valorização $Y$-ádica em $K(Y)$. 
As extensões de um anel $A$, centradas numa valorização $v$ tal que $A \subseteq \mathscr{O}_{v}$, são extensões de $A$ obtidas adicionando um número finito de elementos de $\mathscr{O}_{v}$ a $A$, e depois localizando este novo anel na sua interseção com $m_{v}$. Tais tipos de extensões serão ferramentas para lidar com a uniformização local, que será o tema do próximo capítulo.

Assumiremos aqui que o leitor tenha um pouco de familiaridade com localizações. Usaremos extensões e contrações de ideais primos numa localização, localização comuta com quociente e localização de localização. Esta última chameremos de propriedade básica de localização. Recomendamos, para leitura sobre o tema, o capítulo 6 de [R].

\subsection{Centralização}

Sejam $L$ um corpo e $A$ um subanel de $L$. Dizemos que uma valorização $v$ em $L$ tem centro em $A$ se $A \subseteq \mathscr{O}_{v}$. Neste caso, o centro de $v$ em $A$ é o ideal primo $A \cap m_{v} \operatorname{de} A$. Centralizar $A$ em $v$ é tomar o anel local $A_{v}:=A_{A \cap m_{v}}$. Observe que o centro $A_{v} \cap m_{v}$, de $v$ em $A_{v}$, contém $A \cap m_{v}$, e $\operatorname{logo} A_{v} \cap m_{v}$ é o ideal maximal de $A_{v}$.

Exemplo 4.1. Considere a valorização $v$ do Exemplo 3.11. Se $A:=K[X, Y]$, então o centro de $v$ em $A$ é $(X, Y)$ e $A_{v}=K[X, Y]_{(X, Y)}$.

Observação 4.2. Observe que o exemplo acima é a versão local do caso da variedade algébrica $X=\operatorname{Spec}(A)=\mathbb{A}^{n}$. Neste caso $v$ é centrada na "origem"de $X$ e portanto $v$ tem centro regular em $X$.

Exemplo 4.3. Considere a valorização $v$ do Exemplo 3.12. Se $A=K[x, y]$, então o centro de $v$ em $A$ é $(x, y)$ e $A_{v}=K[x, y]_{(x, y)}$.

Observação 4.4. Observe que o exemplo acima é a versão local do caso em que $X$ é a curva cuspidal e $v$ é centrada na "origem"de $X$. Portanto $v$ tem centro singular em $X$. 
A proposição abaixo relaciona centralizações em diferentes valorizações.

Proposição 4.5. Sejam $L$ um corpo, $v$ e $v^{\prime}$ valorizações em $L$ com $\mathscr{O}_{v} \subseteq \mathscr{O}_{v^{\prime}}$, e $A$ e $A^{\prime}$ subanéis de $\mathscr{O}_{v}$ com $A \subseteq A^{\prime}$. Valem as seguintes propriedades:

(i) $A \subseteq A_{v} \subseteq A_{v}^{\prime}$.

(ii) $A_{v} \subseteq A_{v^{\prime}}=\left(A_{v}\right)_{v^{\prime}}$.

(iii) $\left(A_{v}\right)_{v}=A_{v}$. Em particular, $\left(\mathscr{O}_{v}\right)_{v}=\mathscr{O}_{v}$.

(iv) Se $I$ é um conjunto de geradores para $A \cap m_{v}$, então também é para $A_{v} \cap m_{v}$.

Demonstração. (i) Segue do fato de que $A$ e $A^{\prime}$ são domínios. (ii) A inclusão segue de $m_{v^{\prime}} \subseteq m_{v}$, e a igualdade de propriedade básica de localização. (iii) Propriedade básica de localização. (iv) Segue de $A_{v} \cap m_{v}=\left(A \cap m_{v}\right) A_{v}$.

Os itens i, ii e iii da proposição acima não serão mencionados quando os utilizarmos.

Para as próximas seções deste capítulo, vamos assumir a seguinte configuração: $L$ um corpo, $A$ um subanel de $L, v$ uma valorização que tem centro em $A$ e $v=v^{\prime} \circ \bar{v}$ uma decomposição de $v$. Observe que $v^{\prime}$ tem centro em $A$.

Lembre também que $\left(\mathscr{O}_{\bar{v}}, m_{\bar{v}}\right)$ é o anel de valorização $\left(\mathscr{O}_{v} / m_{v^{\prime}}, m_{v} / m_{v^{\prime}}\right)$ de $\bar{L}_{v^{\prime}}=\mathscr{O}_{v^{\prime}} / m_{v^{\prime}}$. Para qualquer subanel $A^{\prime}$ de $\mathscr{O}_{v}$, denotaremos por $\overline{A^{\prime}}$ o subanel $A^{\prime} / A^{\prime} \cap m_{v}$ de $\mathscr{O}_{\bar{v}}$.

\subsection{Extensões centradas em valorizações}

Uma extensão centrada de $A$ em $v$ é uma extensão de $A$ do tipo $A\left[a_{1}, \cdots, a_{r}\right]_{v}$, com $a_{1}, \cdots, a_{r} \in \mathscr{O}_{v}$. Dizemos apenas que $A\left[a_{1}, \cdots, a_{r}\right]_{v}$ é uma extensão centrada de $(A, v)$. Observe que se $A^{\prime}$ é uma extensão centrada de $(A, v)$, então $v$ tem centro em $A^{\prime}$.

Proposição 4.6. Sejam $a_{1}, a_{2} \in \mathscr{O}_{v}$. Então $A\left[a_{1}, a_{2}\right]_{v}=A\left[a_{1}\right]_{v}\left[a_{2}\right]_{v}$.

Demonstração. Como $A \subseteq A\left[a_{1}\right]_{v}\left[a_{2}\right]_{v}$ e $a_{1}, a_{2} \in A\left[a_{1}\right]_{v}\left[a_{2}\right]_{v}$, temos $A\left[a_{1}, a_{2}\right]_{v}=\left(A\left[a_{1}\right]_{v}\left[a_{2}\right]_{v}\right)_{v}=$ $A\left[a_{1}\right]_{v}\left[a_{2}\right]_{v}$. Analogamente, como $A\left[a_{1}\right]_{v} \subseteq A\left[a_{1}, a_{2}\right]_{v}$ e $a_{2} \in A\left[a_{1}, a_{2}\right]_{v}$, temos a inclusão contrária.

É consequência da proposição acima que, se $A^{\prime}$ é uma extensão centrada de $(A, v)$ e $A^{\prime \prime}$ uma extensão centrada de $\left(A^{\prime}, v\right)$, então $A^{\prime \prime}$ é uma extensão centrada de $(A, v)$.

Em relação à decomposição de $v$, podemos considerar extensões centradas de $\left(A, v^{\prime}\right) \mathrm{e}$ $(\bar{A}, \bar{v})$. Abaixo, relacionaremos extensões centradas destes com extensões centradas de $(A, v)$.

\subsubsection{Caso $v^{\prime}$}

Lema 4.7. Se $a \in \mathscr{O}_{v}^{\times}$, então $A[a]_{v}=A\left[a^{-1}\right]_{v}$. 
Demonstração. Como $a$ é inversível em $\mathscr{O}_{v}$, temos $a \in A[a] \backslash A[a] \cap m_{v}$, e assim $a^{-1} \in A[a]_{v}=$ $A[a]_{A[a] \cap m_{v}}$. Logo $A\left[a^{-1}\right] \subseteq A[a]_{v}$, que implica $A\left[a^{-1}\right]_{v} \subseteq A[a]_{v}$. Analogamente, temos a inclusão oposta.

Proposição 4.8. Dados $b_{1}, \cdots, b_{r} \in \mathscr{O}_{v^{\prime}}$, existem $a_{1}, \cdots, a_{r} \in \mathscr{O}_{v}$ tais que $A\left[a_{1}, \cdots, a_{r}\right]_{v^{\prime}}=$ $A\left[b_{1}, \cdots, b_{r}\right]_{v^{\prime}}$.

O diagrama abaixo ilustra o resultado:

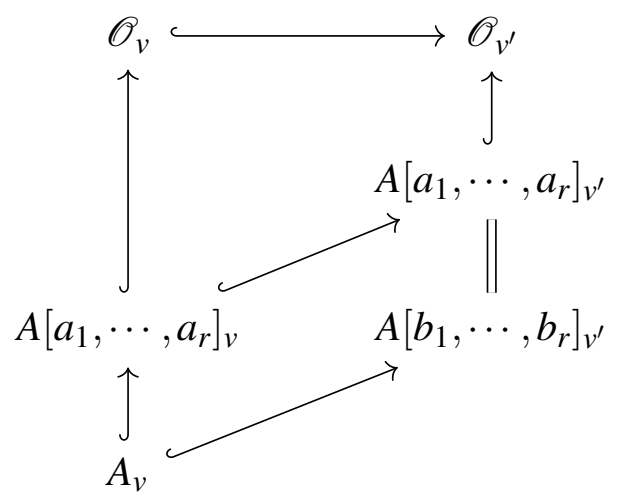

Demonstração. Façamos indução em $r$. Se $r=1$, temos duas possibilidades: se $b_{1} \in \mathscr{O}_{v}$, tomamos $a_{1}=b_{1}$ e o resultado segue. Se $b_{1} \in \mathscr{O}_{v^{\prime}} \backslash \mathscr{O}_{v}$, temos $b_{1}^{-1} \in \mathscr{O}_{v}$. Tomemos $a_{1}=b_{1}^{-1}$. Já que também $b_{1}^{-1} \in \mathscr{O}_{v^{\prime}}, b_{1}$ é inversível em $\mathscr{O}_{v^{\prime}}$, e o lema acima nos dá $A\left[a_{1}\right]_{v^{\prime}}=A\left[b_{1}^{-1}\right]_{v^{\prime}}=$ $A\left[b_{1}\right]_{v^{\prime}}$. Assumimos agora, por hipótese de indução, que o resultado é válido para $r=k$. Para $r=k+1$, temos $A\left[b_{1}, \cdots, b_{k}, b_{k+1}\right]_{v^{\prime}}=A\left[b_{1}, \cdots, b_{k}\right]_{v^{\prime}}\left[b_{k+1}\right]_{v^{\prime}}=A\left[a_{1}, \cdots, a_{k}\right]_{v^{\prime}}\left[b_{k+1}\right]_{v^{\prime}}$, para alguns $a_{1}, \cdots, a_{k} \in \mathscr{O}_{v}$, pela hipótese de indução. Se $b_{k+1} \in \mathscr{O}_{v}$, tome $a_{k+1}=b_{k+1}$. Caso contrário, tome $a_{k+1}=b_{k+1}^{-1}$, e o resultado segue usando o lema.

Observação 4.9. Observe que se $a \in A_{v^{\prime}}$, então $A[a]_{v^{\prime}}=A_{v^{\prime}}[a]_{v^{\prime}}=A_{v^{\prime}}$.

\subsubsection{Caso $\bar{v}$}

Lema 4.10. (i) $\overline{A_{v}}=\bar{A}_{\bar{v}}$.

(ii) Se $\xi \in \mathscr{O}_{\bar{v}} \cap \overline{A_{v^{\prime}}}$, então existe $a \in \mathscr{O}_{v} \cap A_{v^{\prime}}$ tal que $\bar{a}=\xi$.

Demonstração. (i) Localização comuta com quociente. (ii) como $\xi \in \overline{A_{v^{\prime}}}$, existe $a \in A_{v^{\prime}}$ tal que $\bar{a}=\xi$. Como $\bar{a} \in \mathscr{O}_{\bar{v}}=\overline{\mathscr{O}_{v}}$ e $m_{v^{\prime}} \subset \mathscr{O}_{v}$, temos $a \in \mathscr{O}_{v}$.

Proposição 4.11. Se $\bar{b}_{1}, \cdots, \bar{b}_{r} \in \mathscr{O}_{\bar{v}} \cap \overline{A_{v^{\prime}}}$, então existem $a_{1}, \cdots, a_{r} \in \mathscr{O}_{v} \cap A_{v^{\prime}}$ tais que $\overline{A\left[a_{1}, \cdots, a_{r}\right]_{v}}=$ $\bar{A}\left[\bar{b}_{1}, \cdots, \bar{b}_{r}\right]_{\bar{v}}$.

Como no caso $v^{\prime}$, temos o diagrama: 


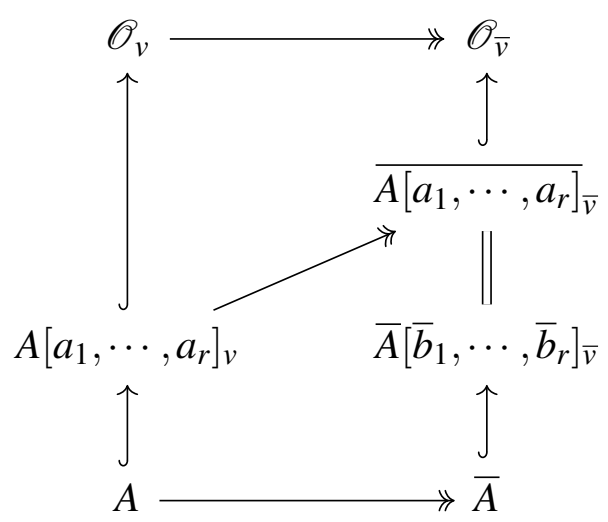

Demonstração. Pelo item (ii) do lema, existem $a_{1}, \cdots, a_{r} \in \mathscr{O}_{v} \cap A_{v^{\prime}}$ tais que $\overline{b_{i}}=\overline{a_{i}}$, e pelo item (i) temos $\overline{A\left[a_{1}, \cdots, a_{r}\right]_{v}}=\overline{A\left[a_{1}, \cdots, a_{r}\right]_{\bar{v}}}=\bar{A}\left[\overline{a_{1}}, \cdots, \bar{a}_{r}\right]_{\bar{v}}=\bar{A}\left[\bar{b}_{1}, \cdots, \bar{b}_{r}\right]_{\bar{v}}$.

Observação 4.12. Observe que se $a \in m_{v^{\prime}}$, então $\bar{a}=0$, e portanto $\bar{A}[\bar{a}]_{\bar{v}}=\bar{A}_{\bar{v}}$.

\subsection{Centros finitamente gerados}

Nesta seção assumiremos que $A$ é noetheriano, e assim serão noetherianos os anéis obtidos, a partir de $A$, por quocientes, localizações e extensões centradas (devido também ao teorema da base de Hilbert).

Lema 4.13. Seja $a \in A \backslash A \cap m_{v}$. Se $b_{1}, \cdots, b_{r}$ geram $A \cap m_{v^{\prime}}$, então $\frac{b_{1}}{a}, \cdots, \frac{b_{k}}{a}, b_{k+1}, \cdots, b_{r}$ geram $A\left[\frac{b_{1}}{a}, \cdots, \frac{b_{k}}{a}\right] \cap m_{v^{\prime}}\left(\mathrm{e} A\left[\frac{b_{1}}{a}, \cdots, \frac{b_{k}}{a}\right]_{v} \cap m_{v}^{\prime}\right.$, devido ao item (iv) da proposição 3.1), para todo $k=1, \cdots, r$.

Demonstração. Como $a \notin m_{v^{\prime}}$, temos $a^{-1} \in \mathscr{O}_{v^{\prime}}$ e, consequentemente, $\frac{b_{i}}{a} \in m_{v^{\prime}}$ para $i=1, \cdots, r$. É fácil ver que $\frac{b_{1}}{a}, \cdots, \frac{b_{k}}{a}, b_{1}, \cdots, b_{r}$ geram $A\left[\frac{b_{1}}{a}, \cdots, \frac{b_{k}}{a}\right] \cap m_{v}^{\prime}$, e como $a \in A, \frac{b_{1}}{a}, \cdots, \frac{b_{k}}{a}, b_{k+1}, \cdots, b_{r}$ geram $A\left[\frac{b_{1}}{a}, \cdots, \frac{b_{k}}{a}\right] \cap m_{v^{\prime}}$.

Teorema 4.14. Se $A_{v^{\prime}}$ e $\bar{A}_{\bar{v}}$ são regulares, então existe uma extensão centrada de $(A, v)$ regular.

Demonstração. O nosso plano é entrar na situação da Proposição 2.3. Vamos encontrar uma extensão centrada $A^{\prime}=A\left[a_{1}, \cdots, a_{t}\right]_{v}$ de $(A, v)$, com $A_{v^{\prime}}^{\prime}=A_{v^{\prime}}$ e ${\overline{A^{\prime}}}_{\bar{v}}=\bar{A}_{\bar{v}}$, tal que $\mu\left(A^{\prime} \cap m_{v^{\prime}}\right) \leq$ $\operatorname{dim} A_{v^{\prime}}$, e daí concluir que $A^{\prime}$ é regular. Para chegarmos na situação desejada, basta que $a_{1}, \cdots, a_{t} \in A_{v^{\prime}} \cap m_{v^{\prime}}$ e $\mu\left(A^{\prime} \cap m_{v^{\prime}}\right) \leq \operatorname{dim} A_{v^{\prime}}$, pois neste caso $\bar{A}\left[\overline{a_{1}}, \cdots, \bar{a}_{t}\right]_{\bar{v}}=\bar{A}_{\bar{v}}$ devido a $\overline{a_{i}}=\overline{0}$, e $A\left[a_{1}, \cdots, a_{t}\right]_{v^{\prime}}=A_{v^{\prime}}$ devido a $a_{i} \in A_{v^{\prime}}$.

Sejam $r=\operatorname{dim} A_{v^{\prime}}$ e $b_{1}, \cdots, b_{r} \in A \cap m_{v^{\prime}}$ geradores de $A_{v^{\prime}} \cap m_{v^{\prime}}$ (veja na seção 1.1). Se $b_{1}, \cdots, b_{r}$ geram $A \cap m_{v^{\prime}}$, basta tomar $A^{\prime}=A_{v}$ e o objetivo está alcançado. Caso não gerem, adicionamos $c_{1}, \cdots, c_{s} \in A \cap m_{v^{\prime}}$ ao nosso conjunto para gerar $A \cap m_{v^{\prime}}$. Existe (também veja na seção 1.1) $a \in A \backslash A \cap m_{v^{\prime}}$ tal que $a c_{1} \in\left(b_{1}, \cdots, b_{r}\right) \subset A$, e assim $c_{1} \in\left(\frac{b_{1}}{a}, \cdots, \frac{b_{r}}{a}\right) \subset A\left[\frac{b_{1}}{a}, \cdots, \frac{b_{r}}{a}\right]$. Pelo lema 3.4, $\frac{b_{1}}{a}, \cdots, \frac{b_{r}}{a}, c_{2}, \cdots, c_{s}$ geram $A\left[\frac{b_{1}}{a}, \cdots, \frac{b_{r}}{a}\right] \cap m_{v^{\prime}}$. Temos também $\frac{b_{1}}{a}, \cdots, \frac{b_{r}}{a} \subset A_{v^{\prime}} \cap m_{v^{\prime}}$, 
e estes geram $A_{v^{\prime}} \cap m_{v^{\prime}}$ em $A_{v^{\prime}}$. Estamos novamente como na configuração inicial e diminuímos em 1 o nosso conjunto de geradores. Repetindo este processo mais $s-1$ vezes, e por fim centralizando em $v$, (e usando a parte centralizada do lema 3.4), obtemos o resultado. 



\section{UNIFORMIZAÇÃO LOCAL}

Daremos agora a definição de uniformização local, que é uma parte do método de Zariski para provar resolução de singularidades em variedades algébricas, e o principal tema desta dissertação. O nosso objetivo é mostrar que provar uniformização local para uma classe de objetos e valorizações, pode ser reduzido a provar apenas os casos de valorizações de posto um.

\subsection{Uniformização local}

Seja $L \mid K$ uma extensão de corpos. Se $L=K\left(x_{1}, \cdots, x_{r}\right)$ para alguns $x_{1}, \cdots, x_{r} \in L$, dizemos que $L$ é um corpo de funções algébricas sobre $K$, ou, de forma mais curta, $L \mid K$ é um corpo de funções. Um modelo para um corpo de funções $L \mid K$ é uma $K$-álgebra finitamente gerada com corpo de frações igual a $L$. Como o corpo de frações de uma $K$-álgebra finitamente gerada que é domínio de integridade é um corpo de funções sobre $K$, chamamos qualquer $K$-álgebra deste tipo de modelo sobre $K$, sem mencionar a extenção de corpos.

Sejam $L \mid K$ um corpo de funções e $A$ um modelo para $L \mid K$. Se $v$ é uma valorização em $L$ que tem centro em $A$, dizemos que $v$ tem centro regular em $A$ se o anel $A_{v}$ é regular. Um blowing up local de $A$, com respeito a $v$, é uma extensão de $A$ do tipo $A^{\prime}=A\left[a_{1}, \cdots, a_{r}\right]$, com $a_{1}, \cdots, a_{r} \in \mathscr{O}_{v}$. Dizemos simplesmente que $A^{\prime}$ é um blowing up local de $(A, v)$. Observe que $A^{\prime}$ também é um modelo para $L \mid K$ e $v$ tem centro em $A^{\prime}$.

O problema da uniformização local é o seguinte:

(UL) Sejam $L \mid K$ um corpo de funções, $A$ um modelo para $L \mid K$ e $v$ uma valorização em $L$ que tem centro em A. Existe um blowing up local $A^{\prime}$ de $(A, v)$ cujo centro de $v$ em $A^{\prime}$ é regular?

Dizemos que o par $(A, v)$ admite uniformização local se o problema acima pode ser resolvido. 


\subsection{Uniformização local e decomposição de valorizações}

Comecemos com uma convenção. Sejam $L \mid K$ um corpo, $A$ um modelo para $L \mid K$, e $v$ uma valorização numa extensão de corpos de $L$ com centro em $A$. Perguntar sobre blowing up local de $(A, v)$, significa considerar a restrição de $v$ a $\operatorname{Frac}(A)$. O mesmo vale para uniformização local.

Seja $v=v^{\prime} \circ \bar{v}$ uma decomposição de $v$. Observe que $\bar{A}=A /\left(A \cap m_{v^{\prime}}\right)$ é modelo sobre $K$, e que $\operatorname{Frac}(\bar{A})=\overline{A_{v^{\prime}}}=A_{v^{\prime}} /\left(A_{v^{\prime}} \cap m_{v^{\prime}}\right)$ (basta comutar localização com quociente).

Para o teorema abaixo, considere os objetos já dados e o conjunto $\mathscr{M}_{\bar{v}}$ de todos os modelos em que $\bar{v}$ tem centro.

Teorema 5.1. Se todo par $(\bar{B}, \bar{v})$, onde $\bar{B} \in \mathscr{M}_{\bar{v}}$, e $\left(A, v^{\prime}\right)$ admitem uniformização local, então $(A, v)$ admite uniformização local.

Demonstração. Basta mostrarmos que existe um blowing up local $A^{\prime}$ de $(A, v)$ tal que $A_{v^{\prime}}^{\prime}$ e $\overline{A^{\prime}} \bar{v}$ são regulares, devido ao Teorema 4.14.

Como $\left(A, v^{\prime}\right)$ admite uniformização local, existe uma extensão centrada $A\left[a_{1}, \cdots, a_{r}\right]_{v^{\prime}}$ de $\left(A, v^{\prime}\right)$ regular. Pela Proposição $4.8, a_{1}, \cdots, a_{r}$ podem ser tomados em $\mathscr{O}_{v}$. Trocamos então $A$ por $A^{\prime \prime}:=A\left[a_{1}, \cdots, a_{r}\right]$. Como $\left(\overline{A^{\prime \prime}}, \bar{v}\right)$ admite uniformização local, existe uma extensão centrada $\overline{A^{\prime \prime}}\left[\overline{b_{1}}, \cdots, \overline{b_{s}}\right]_{\bar{v}}$ de $\left(\overline{A^{\prime \prime}}, \bar{v}\right)$ regular. Observe que $b_{1}, \cdots, b_{s} \in A_{v^{\prime}} \cap \mathscr{O}_{v}$. Trocamos agora $A^{\prime \prime}$ por $A^{\prime}:=A^{\prime \prime}\left[b_{1}, \cdots, b_{s}\right]$, que é um blowing up local de $(A, v)$. Este $A^{\prime}$ é o blowing up local de $(A, v)$ que procurávamos. Como $b_{1}, \cdots, b_{s} \in A_{v^{\prime}} \subseteq A_{v^{\prime}}^{\prime \prime}$, temos a igualdade $A_{v^{\prime}}^{\prime}=A^{\prime \prime}\left[b_{1}, \cdots, b_{s}\right]_{v^{\prime}}=$ $A_{v^{\prime}}^{\prime \prime}\left[b_{1}, \cdots, b_{s}\right]_{v^{\prime}}=A_{v^{\prime}}^{\prime \prime}=A\left[a_{1}, \cdots, a_{r}\right]_{v^{\prime}}$, e logo $A_{v^{\prime}}^{\prime}$ é regular. E também $\overline{A^{\prime}} \bar{v}=\overline{A^{\prime \prime}}\left[\overline{b_{1}}, \cdots, \overline{b_{s}}\right]_{\bar{v}}$ é regular. Portanto o resultado está provado.

Observe que, se $v$ tem posto finito, podemos decompor também $\bar{v}$ e $v^{\prime}$ e reduzir o problema da uniformização local para valorizações de posto cada vez menores, e seguindo assim, reduzir o problema para valorizações de posto um.

\subsection{Dimensão e posto}

Antes de enunciarmos o resultado final e concluirmos a dissertação, precisamos garantir que as valorizações que iremos lidar são de posto finito. Este é o objetivo desta seção.

Seja $L \mid K$ um corpo de funções. Dizemos que $v$ é valorização em $L \mid K$ se $v$ é uma valorização em $L$ trivial em $K$. É consequência da desigualdade da dimensão ([EP], Corolário 3.4.4) que, se $v$ é trivial em $K$, então o posto de $v$ é menor ou igual ao grau de transcendência de $L \mid K$. Como o grau de transcendência de $L \mid K$ é finito, $v$ tem posto finito. Como todo modelo sobre $K$ contém $K$, todas valorizações que lidamos são triviais em $K$, pois têm centros em modelos, e portanto de posto finito. 
Se $A$ é um modelo para $L \mid K$ que tem centro em $v$ e $v=v^{\prime} \circ \bar{v}$ uma decomposição de $v$, então, pela Observação 3.13, a restrição de $\bar{v}$ ao corpo de frações de $\bar{A}$ tem posto menor ou igual ao de $\bar{v}$. Isto nos garante que, se $v=v^{\prime} \circ \bar{v}$ é uma decomposição não trivial, então quando perguntarmos sobre blowing ups locais de $\left(A, v^{\prime}\right)$ e $(\bar{A}, \bar{v})$, estamos lidando com valorizações de posto menor que o posto de $v$.

A desigualdade da dimensão também nos dá que o grau de transcendência de $\bar{L}_{v} \mid K$ é menor ou igual ao grau de transcendência de $L \mid K$. Então, se a decomposição não é trivial, o grau de transcendência de $\overline{A_{v^{\prime}}} \mid K$ é menor que o grau de transcendência de $L \mid K$, onde $\overline{A_{v^{\prime}}}$ é o corpo de frações de $\bar{A}$.

\subsection{Redução a valorizações de posto um}

Esta seção conclui a dissertação, que tem por objetivo provar que o problema da uniformização local pode ser reduzido a provar uniformização local para os casos que envolvem apenas valorizações de posto um. Tal resultado já foi provado na seção 5.2, mas agora enunciaremos o problema na forma em que é mais aplicado.

Sejam $K$ um corpo e $\mathscr{M}$ a categoria de todas as $K$-álgebras. Seja $T$ um subconjunto de modelos de $M$. Consideramos a subcategoria $\mathscr{M}_{T}$ de $\mathscr{M}$ que é o fecho de $T$ no seguinte sentido: $T \subseteq \mathscr{M}_{T}$, e $\mathscr{M}_{T}$ é fechado para localizações, quocientes por primos e blowing up locais. Além disso, toda subcategoria de $\mathscr{M}$ que contém $T$ e é fechada neste sentido, contém $\mathscr{M}_{T}$.

Teorema 5.2. Se todo $\operatorname{par}(A, w)$, onde $A \in \mathscr{M}_{T}$ é um modelo e $w$ uma valorização de posto 1 , admite uniformização local, então todo par $(A, v)$ admite uniformização local, onde $A \in \mathscr{M}_{T}$ é um modelo.

Demonstração. Por indução no posto de $v$. Se o posto de $v$ é 1, está provado por hipótese. Se o resultado for válido para todas valorizações de posto $k<n$, então, se $v$ tem posto $n>1$, podemos decompor $v$ em duas valorizações de posto menor, e o resultado segue do Teorema 5.1.

Veja que temos bastante liberdade para $T$. A escolha de $T$ determina o caso específico ao qual se quer trabalhar. Por exemplo, se estamos interessados no caso em que $T$ é o conjunto de todos os modelos possíveis para um corpo de funções $L \mid K$, então nos basta provar que todos os pares de modelos e valorizações de posto um, de $L \mid K$ e corpos de funções com grau de transcendência menor que de $L \mid K$, admitem uniformização local, para se ter uniformização local para todos os modelos e valorizações em $L \mid K$. 

[A1] S. Abhyankar, Local uniformization on algebraic surfaces over ground Fields of characteristic $p \neq 0$, Ann. of Math. 63 (1956), 491-526.

[A2] S. Abhyankar, Resolution of Singularities of Embedded Algebraic Surfaces, Pure and Applied Mathematics 24, Academic Press, New York and London 1966.

[C1] Cossart V. e Piltant O., Resolution of singularities of threefolds in positive characteristic I. Reduction to local uniformization on Artin-Schreier and purely inseparable coverings, J. Algebra 320 (2008), no. 3, 1051-1082.

[C2] Cossart V. e Piltant O., Resolution of singularities of threefolds in positive characteristic II, J. Algebra 321 (2009), no. 7, 1836-1976.

[C] S. D. Cutkosky, Resolution of Singularities. Graduate Studies in Mathematics, Volume 63, American Mathematical Society, Providence, Rhode Island 2004.

[EP] A. J. Engler e A. Prestel, Valued Fields, Springer Monographs in Mathematics, SpringerVerlag, Berlin 2005.

$[\mathrm{H}] \mathrm{H}$. Hironaka, Resolution of singularities of an algebraic variety over a Field of characteristic zero. Ann. of Math. 79 (1964), 109-326.

[KK1] H. Knaf e F.-V. Kuhlmann, Abhyankar places admit local uniformisation in any characteristic. Ann. Sci. Éc. Norm. Supér. (4) 38 no. 6 (2005), 833-846.

[KK2] H. Knaf e F.-V. Kuhlmann, Every place admits local uniformization in a finite extension of the function field, Adv. Math. 221 no. 2 (2009), 428-453. [N] M. Nagata, Local Rings, Robert E. Krieger Publishing Company, Huntington, New York 1975.

[NS] J. Novacoski e M. Spivakovsky, Reduction of local uniformization to the rank one case, Valuation Theory in Interaction, EMS Series of Congress Reports (2014), 404-431.

[R] M. Reid, Undergraduate commutative algebra, Student Texts, London Mathematical Society, Cambrige University Press, 29, 1995.

[S] J. Serre, Local Algebra, Springer Monographs in Mathematics, Springer-Verlag, Berlin 2000. [Sp] M. Spivakovsky, Resolution of singularities I: the local uniformization theorem for quasi-excellent noetherian schemes, preprint.

[T1] Teissier B., Valuations, Deformations and Toric Geometry, in Valuation theory and its applications, Vol. II, (Saskatoon, SK, 1999), Fields Inst. Commun. 33 (2003), 361-459.

[T2] B. Teissier, Overweight deformations of affine toric varieties and local uniformization, Valuation Theory in Interaction, EMS Series of Congress Reports (2014), 474-565. [V] Vaquié, M., Valuations and local uniformization, Singularity theory and its applications, 477-527, Adv. Stud. Pure Math., 43, Math. Soc. Japan, Tokyo, 2006. 
[Z] O. Zariski, Local uniformization theorem on algebraic varieties, Ann. of Math. 41 (1940), 852-896. 